\title{
Regularization of the second-order gravitational perturbations produced by a compact object
}

\author{
Eran Rosenthal \\ Department of Physics, University of Guelph, \\ Guelph, Ontario N1G 2W1, Canada
}

(Dated: December 30, 2018)

\begin{abstract}
The equations for the second-order gravitational perturbations produced by a compact-object have highly singular source terms at the point particle limit. At this limit the standard retarded solutions to these equations are ill-defined. Here we construct well-defined and physically meaningful solutions to these equations. These solutions are important for practical calculations: the planned gravitational-wave detector LISA requires preparation of waveform templates for the expected gravitational-waves. Construction of templates with desired accuracy for extreme mass ratio binaries, in which a compact-object inspirals towards a supermassive black-hole, requires calculation of the second-order gravitational perturbations produced by the compact-object.
\end{abstract}


In an extreme mass-ratio binary, where a compact-object $(\mathrm{CO})$ of mass $\mu$ (e.g. a neutron star or a small black hole) orbits a supermassive black hole of mass $M$, the interaction between the $\mathrm{CO}$ and the gravitational perturbations that it produces exerts a force on the CO. This phenomenon of gravitational self-force (GSF) is responsible for the CO's gradual inspiral towards the supermassive black-hole. The small parameter $\mu / M$ allows treating the spacetime metric and the CO's orbit perturbatively. At the leading order of this approximation the orbit traces a geodesic in the background spacetime of the supermassive black-hole [1]. At the next order, the CO's interaction with the $O(\mu)$ first-order metric perturbations (FOMP) produces a first-order GSF that accelerates the CO in the background spacetime [2, 3]. At the next order, the interaction with the $O\left(\mu^{2}\right)$ second-order metric perturbations (SOMP) produces a second-order GSF, and so on.

Extreme mass-ratio binaries (e.g., $M / \mu=10^{5}$ ) are valuable sources of gravitational waves (GW) that could be detected by the planned Laser Interferometer Space Antenna (LISA) 4]. Detection of these sources and determination of their parameters using matched-filtering data-analysis techniques requires preparation of gravitational waveform templates for the expected GW. Here one of the main challenges is to calculate the GW's accumulating phase, e.g. a wave-train from one year of inspiral can contain about $10^{5} \mathrm{GW}$ cycles [5]. Successful determination of the binary parameters using matched-filtering techniques often requires GW templates in which the phase error is less than one radian over a year 6] .

The following simple scaling argument reveals how the phase scales with $\mu$, and allows us to quantify the desired degree of accuracy for the metric perturbation expansion (see also 7, 8]). For simplicity consider a CO that gradually inspirals between two otherwise circular geodesic orbits in a strong field region of a Schwarzschild black hole. We are interested in estimating the effect of the GSF on the accumulated phase of the emitted GW. Due to the GSF the orbital frequency slowly changes from its value at an initial time, and after time $t$ has elapsed this frequency has shifted by approximately $\dot{\omega} t$ from its initial value, where $\dot{\omega} \equiv \frac{d \omega}{d t}$, and $t$ denotes the elapsed time (from initial time) in Schwarzschild coordinates. We denote $\Delta \phi$ the part of the phase shift of the GW (between two fixed times) which is induced by the shift in the orbital frequency. Recalling that the GW frequency is proportional to the orbital frequency we find that after an inspiral time $\Delta t_{\text {ins }}$, the phase shift $\Delta \phi$ is approximately proportional to $\Delta t_{\text {ins }}^{2} \dot{\omega}$. We shall now find how the quantities in this expression scale with $\mu$. Let us consider first $\dot{\omega}$. We write this quantity as $\dot{\omega}=\frac{d \omega}{d E} \dot{E}$, where $E$ denotes the particle's 
energy per unit mass. Notice that $\frac{d \omega}{d E}$ is independent of $\mu$ (it is obtained from an analysis of a circular geodesic worldline) while $\dot{E}$ is determined from the four-acceleration and therefore depends on $\mu$. The first-order GSF produces the first-order terms in the expansions of $\dot{E}$ and $\dot{\omega}$; these terms are denoted here $\dot{E}_{1}$ and $\dot{\omega}_{1}$, respectively. Since $\dot{E}_{1}$ scales like $\mu$ we find that $\dot{\omega}_{1}$ is $O\left(\mu M^{-3}\right)$. At the leading order the inspiral is driven by the first-order GSF and therefore the inspiral time $\Delta t_{\text {ins }}$ is of the order $\Delta E \dot{E}^{-1}=O\left(M^{2} \mu^{-1}\right)$, where $\Delta E$ is the energy difference between initial and final orbits. After $\Delta t_{\text {ins }}$ the term $\dot{\omega}_{1}$ will give rise to a phase shift of order $\Delta t_{i n s}^{2} \dot{\omega}_{1}=O(M / \mu)$. The second-order GSF produces second-order terms in the expansions of $\dot{E}$, and $\dot{\omega}$. These terms are denoted here $\dot{E}_{2}$ and $\dot{\omega}_{2}$, respectively. Since $\dot{E}_{2}$ scales like $\mu^{2}$ it gives rise to an $\dot{\omega}_{2}$ which is $O\left(\mu^{2} M^{-4}\right)$. After $\Delta t_{i n s}$ the term $\dot{\omega}_{2}$ will produce a phase shift of order $\Delta t_{i n s}^{2} \dot{\omega}_{2}=O\left[(M / \mu)^{0}\right]$. Therefore, a calculation of $\Delta \phi$ to the desired accuracy of order $(M / \mu)^{0}$ (needed for LISA data analysis) requires the calculation of the CO's interaction with its own SOMP.

The challenge of constructing long waveform templates with $O\left[(M / \mu)^{0}\right]$ accuracy provides a practical motivation for the study of SOMP in this article. Furthermore, construction of SOMP will extend the applicability of the perturbative scheme to binaries with smaller $M / \mu$ mass-ratios.

Consider the limit where the spatial dimensions of the $\mathrm{CO}$ approach zero; we shall refer to this limit as the point particle limit (Below we shall make more precise definitions of the $\mathrm{CO}$ and the limiting process that we use.). In this limit the SOMP away from the CO satisfy the following equation

$$
D[l]=\nabla h \nabla h \& h \nabla \nabla h .
$$

Here we used a schematic notation, where the SOMP are denoted $\mu^{2} l, D$ denotes a linear partial differential operator, the retarded FOMP are denoted $\mu h, \nabla$ denotes a covariant derivative with respect to the background metric, and \& denotes "and terms of the form ...". In the point particle limit (certain components of) the FOMP in the Lorenz gauge diverge as $r^{-1}$, where $r$ denotes the spatial distance from the object. Here we encounter a problem, since the $O\left(r^{-1}\right)$ divergent behavior of $h$ implies that the source term of Eq. (1D) diverges as $r^{-4}$. A naive attempt to construct the standard retarded solution to Eq. (ID) by imposing Lorenz gauge conditions on $l$, and then integrating the resultant wave equation using the retarded Green's function, results in an integral that diverges at every point in spacetime. A similar problem in a scalar toy-model has recently been studied [9]. In this 
article we develop a regularization method for the construction of well-defined and physically meaningful solutions to Eq. (11).

We should mention here another regularization problem coming from infinity. For an infinitely long worldline, the leading asymptotic behavior of the source term in Eq. (II) is $O\left(r^{-2}\right)$. This behavior at infinity renders the retarded solution $l$ divergent ${ }^{1}$. This issue, however, lies outside the scope of this article. Here we assume that a regularization at infinity had been carried out.

For simplicity we choose the $\mathrm{CO}$ to be a Schwarzschild black hole, which moves in a vacuum background geometry characterized by length scales that are much larger than $\mu$. Designating the length scales associated with the Riemann curvature tensor of the background geometry with $\left\{\mathcal{R}_{i}\right\}$, and denoting $\mathcal{R}=\min \left\{\mathcal{R}_{i}\right\}$, we express the above restriction as $\mu \ll \mathcal{R}$.

Our analysis is based on the method of matched asymptotic expansions (see e.g. [10, 11]), in which different approximate solutions to Einstein's field equations are obtained in different, overlapping, regions of spacetime. These solutions are then matched in their common region of validity. Consider the following decomposition of spacetime into an internal zone that lies within a worldtube which surrounds the black hole and extends out to $r=r_{I}(\mathcal{R})$ such that $r_{I} \ll \mathcal{R}$, and an external zone that lies outside another worldtube at $r=r_{E}(\mu)$, such that $\mu \ll r_{E}$. The interior of this inner worldtube is denoted $S$. By virtue of $\mu \ll \mathcal{R}$ we choose $r_{E}$ to be much smaller than $r_{I}$ such that there is an overlap between the above mentioned regions in $r_{E}<r<r_{I}$. We refer to this overlap region as the buffer zone (in the buffer zone $r$ can be of order $\sqrt{\mu \mathcal{R}}$ ).

In the external zone we decompose the full spacetime metric $g_{\mu \nu}^{f u l l}$ into a background metric $g_{\mu \nu}$ (e.g. a spacetime of a supermassive black hole), and a sequence of perturbations that are produced by the Schwarzschild black hole (with mass $\mu$ ), reading

$$
g_{\mu \nu}^{f u l l}(x)=g_{\mu \nu}(x)+\mu h_{\mu \nu}(x)+\mu^{2} l_{\mu \nu}(x)+O\left(\mu^{3}\right) .
$$

\footnotetext{
${ }^{1}$ Asymptoticly $h$ has a form of a gravitational wave. Therefore the dominant terms in an asymptotic expansion of $\nabla h$ decay like $r^{-1}$. This implies that the source term in Eq. (1) is asymptotically $O\left(r^{-2}\right)$. Notice that this source term has a static $O\left(r^{-2}\right)$ component which does not vanish after averaging over time. An attempt to construct the retarded solution to Eq. (11) with this static term present yields a divergent expression (even if we resolve the difficulty with the singularity in the vicinity of the worldline). Ori has recently suggested a resolution to this problem [15].
} 
Here the dependence on $\mu$ is only through the explicit powers $\mu^{i}$. Throughout we use the background metric $g_{\mu \nu}$ to raise and lower tensor indices, and to evaluate covariant derivatives. Substituting expansion (2) into Einstein's field equations in vacuum yields linear perturbation equations for $h_{\mu \nu}$ and $l_{\mu \nu}$. These equations are valid for $x \notin S$. Here we shall be interested in the limit $\mu \rightarrow 0$. Note that this limit has no effect on the forms of the perturbations equations. Yet their region of validity is affected since we let $r_{E}(\mu) \rightarrow 0$ as $\mu \rightarrow 0$. In this limit the perturbation equations read

$$
\begin{aligned}
& D_{\mu \nu}[\bar{h}]=0, x \notin z(\tau), \\
& D_{\mu \nu}[\bar{l}]=S_{\mu \nu}[\bar{h}], x \notin z(\tau) .
\end{aligned}
$$

Here $z(\tau)$ is a timelike worldline, where $\tau$ denotes proper time with respect to the background metric. An overbar denotes the trace-reversal operator $\bar{h}_{\mu \nu}=h_{\mu \nu}-(1 / 2) g_{\mu \nu} h_{\alpha}{ }^{\alpha}$; for brevity we have omitted tensorial indices inside the squared brackets. $D_{\mu \nu}$ and $S_{\mu \nu}$ are obtained from an expansion of the full Ricci tensor. By substituting $g_{\mu \nu}+\delta g_{\mu \nu}$ into the Ricci tensor we obtain $R_{\mu \nu}^{f u l l}=R_{\mu \nu}^{(0)}+R_{\mu \nu}^{(1)}[\delta g]+R_{\mu \nu}^{(2)}[\delta g]+O\left(\delta g^{3}\right)$. (For explicit expressions for the terms in this expansion see e.g. 12]. We adopt the sign convention of this reference for the Riemann tensor). To simplify the notation we denote $\bar{R}_{\mu \nu}^{(1)}[h]$ by $D_{\mu \nu}[\bar{h}]$, and denote $-\bar{R}_{\mu \nu}^{(2)}[h]$ by $S_{\mu \nu}[\bar{h}]$, where $h_{\mu \nu}$ is expressed in terms of $\bar{h}_{\mu \nu}$.

By imposing the Lorenz gauge conditions $\bar{h}_{; \nu}^{\mu \nu}=0$, and matching with the internal-zone solution, D'Eath showed [1] that $h_{\mu \nu}$ is identical to the retarded FOMP produced by a unitmass point particle tracing the worldline $z(\tau)$. At this leading order of approximation $z(\tau)$ is a geodesic of the background spacetime [1], denoted $z_{G}(\tau)$. Substituting $z(\tau)=z_{G}(\tau)$ in Eq. (3) provides us with sufficient accuracy for the calculation of the FOMP. However, to calculate the SOMP we must account for the $O(\mu)$ acceleration of $z(\tau)$ due to the first-order GSF corrections. To account for these corrections we use the gauge dependence of the firstorder GSF [13], and choose a convenient gauge in which the first-order GSF vanishes. In this gauge, the geodesic worldline $z_{G}(\tau)$ is sufficiently accurate for the construction of the SOMP.

To spell out the desired gauge conditions we examine the expression for the $O(\mu)$ acceleration which is produced by the first-order GSF in the Lorenz gauge [14]

$$
a^{\mu}=-\frac{\mu}{2}\left(g^{\mu \nu}+u^{\mu} u^{\nu}\right) u^{\rho} u^{\eta}\left(2 \nabla_{\rho} h_{\eta \nu}^{R}-\nabla_{\nu} h_{\rho \eta}^{R}\right) .
$$


Here all quantities are evaluated on the worldline. We use the Detweiler-Whiting decomposition $h_{\mu \nu}=h_{\mu \nu}^{S}+h_{\mu \nu}^{R}$, where $h_{\mu \nu}^{R}$ and $h_{\mu \nu}^{S}$ are certain regular and singular potentials, respectively (for their definitions and properties see [14]). Consider now a regular gauge transformation generated by a vector field $\xi^{\mu}$. In the new gauge the FOMP, $h_{\mu \nu}^{F}$, are given by $h_{\mu \nu}^{F}=h_{\mu \nu}^{S}+h_{\mu \nu}^{R(F)}$, where $h_{\mu \nu}^{R(F)} \equiv h_{\mu \nu}^{R}+\xi_{\mu ; \nu}+\xi_{\nu ; \mu}$ (The gauge invariance of $h_{\mu \nu}^{S}$ follows from the analysis in Ref. [13].).

Many gauge choices can provide us with the desired requirement $a^{\mu}=0$. For simplicity we impose the following gauge conditions on the worldline:

$$
\left[h_{\mu \nu}^{R(F)}\right]_{z_{G}(\tau)}=0,\left[\nabla_{\rho} h_{\mu \nu}^{R(F)}\right]_{z_{G}(\tau)}=0 .
$$

We shall refer to this gauge as the Fermi gauge. We may now replace the restrictions $x \notin z(\tau)$ with $x \notin z_{G}(\tau)$ in Eqs. (34). Notice that the gauge conditions (6) are specified only on $z_{G}(\tau)$. To complete the gauge construction we adopt an arbitrary continuation of $\xi_{\mu}$ to the entire spacetime (e.g., continuation along future null-cones based on $z_{G}(\tau)$ in a manner that preserves causality ${ }^{2}$ ).

Before solving Eq. (44) we study the singular properties of $S_{\mu \nu}$ near $z_{G}(\tau)$. Consider expanding $S_{\mu \nu}$ in the vicinity of $z_{G}(\tau)$, on a family of hypersurfaces $\tau=$ const that are generated by geodesics that are normal to the worldline. We use Fermi normal coordinates based on $z_{G}(\tau)$, thus obtaining simple expressions. We find that

$$
S_{\mu \nu}(x) \stackrel{*}{=} A_{\mu \nu} r^{-4}+O\left(r^{-2}\right) .
$$

Here $\stackrel{*}{=}$ denotes equality in Fermi normal coordinates. We have $A_{\mu \nu} \equiv 4 u_{\mu} u_{\nu}+7 \eta_{\mu \nu}-$ $14 \Omega_{\mu} \Omega_{\nu}, \eta_{\mu \nu}$ denotes the Minkowski metric, $r=\sqrt{\delta_{a b} x^{a} x^{b}}$, where $x^{a}$ denote the spatial Fermi coordinates $(a, b$ take the values $\{1,2,3\}) ; u^{\mu} \stackrel{*}{=} \delta_{0}^{\mu}$ is a vector field, $\Omega^{a} \stackrel{*}{=} x^{a} / r$, $\Omega^{0} \stackrel{*}{=} 0$, and $\Omega_{\nu} \stackrel{*}{=} g_{\nu \mu} \Omega^{\mu}$. The absence of $O\left(r^{-3}\right)$ terms in Eq. (7) follows from the gauge conditions (6) together with the fact that $z_{G}(\tau)$ is a geodesic worldline.

\footnotetext{
${ }^{2}$ To construct this continuation consider the future null-cones $\Sigma_{\tau}$ emanating from the worldline $z_{G}(\tau)$. Here we focus on a local neighborhood of the worldline in which these null-cones do not intersect each other. For an arbitrary point $z_{G}\left(\tau^{-}\right)$one may choose an arbitrary continuation of $\xi^{\mu}$ on $\Sigma_{\tau^{-}}$, such that $\xi^{\mu}$ decays to zero away from the worldline. In this way the constructed gauge preserve causality in the following sense: The perturbations on the null-cones $\Sigma_{\tau}$ for $\tau \leq \tau^{-}$will remain unchanged if one modifies the worldline for $\tau>\tau^{-}$. Such a modification of the worldline is possible by introducing additional GW that interact with the worldline for $\tau>\tau^{-}$.
} 
To tackle the $r^{-4}$ singularity of $S_{\mu \nu}$ we decompose $\bar{l}_{\mu \nu}$ into two tensor potentials, reading

$$
\bar{l}_{\mu \nu}=\bar{\psi}_{\mu \nu}+\delta \bar{l}_{\mu \nu}
$$

where $\bar{\psi}_{\mu \nu}$ satisfies $D_{\mu \nu}[\bar{\psi}] \stackrel{*}{=} A_{\mu \nu} r^{-4}+O\left(r^{-2}\right)$. Notice that this equation has the same $r^{-4}$ singular source term as Eq. (41). Here, however, we do not impose restrictions on the terms of order $O\left(r^{-2}\right)$. Suppose that we can construct a solution $\bar{\psi}_{\mu \nu}$, then by subtracting $D_{\mu \nu}[\bar{\psi}]$ from both sides of Eq. (41) we obtain

$$
D_{\mu \nu}[\delta \bar{l}]=\delta S_{\mu \nu} \quad, x \notin z_{G}(\tau)
$$

Here $\delta S_{\mu \nu} \equiv S_{\mu \nu}-D_{\mu \nu}[\bar{\psi}]$. By construction $\delta S_{\mu \nu}$ diverges as $r^{-2}$, while $S_{\mu \nu}$ in Eq. (4) diverges as $r^{-4}$. In this sense Eq. (9) is simpler than Eq. (41).

To construct $\bar{\psi}_{\mu \nu}$ we use a linear combination of terms that are quadratic in $\bar{h}_{\mu \nu}^{F}$. Since $\bar{h}_{\mu \nu}^{F}$ diverges as $r^{-1}$ we find that by applying the operator $D_{\mu \nu}$ to terms which are quadratic in $\bar{h}_{\mu \nu}^{F}$ we obtain terms which diverge as $r^{-4}$. First we construct four independent quadratic tensor fields: $\varphi_{\mu \nu}^{A}=\bar{h}_{\mu \rho}^{F \rho} \bar{h}_{\rho \nu}^{F}, \varphi_{\mu \nu}^{B}=\bar{h}^{F \rho}{ }_{\rho} \bar{h}_{\mu \nu}^{F}, \varphi_{\mu \nu}^{C}=\left(\bar{h}^{F \eta \rho} \bar{h}_{\eta \rho}^{F}\right) g_{\mu \nu}, \varphi_{\mu \nu}^{D}=\left(\bar{h}^{F \rho}\right)^{2} g_{\mu \nu}$. Combining these terms we may construct the desired $\bar{\psi}_{\mu \nu}$, which reads

$$
\bar{\psi}_{\mu \nu}=\frac{1}{64}\left[2\left(c_{A} \varphi_{\mu \nu}^{A}+c_{B} \varphi_{\mu \nu}^{B}\right)-7\left(c_{C} \varphi_{\mu \nu}^{C}+c_{D} \varphi_{\mu \nu}^{D}\right)\right]
$$

Here the constants $c_{A}, c_{B}, c_{C}, c_{D}$ must satisfy $c_{A}+c_{B}=1, c_{C}+c_{D}=1$, but are otherwise arbitrary. Roughly speaking, $\bar{\psi}_{\mu \nu}$ captures the asymptotic behavior of the Schwarzschild solution at second order. Due to this property $\bar{\psi}_{\mu \nu}$ is an exact solution to Eq. (4) for the case of a flat background spacetime.

Having constructed $\bar{\psi}_{\mu \nu}$, we now face the problem of solving Eq. (9). For this purpose we invoke a purely second-order gauge transformation of the form $x^{\mu} \rightarrow x^{\mu}-\mu^{2} \xi_{(2)}^{\mu}$ that allows us to impose the Lorenz gauge conditions $\delta \bar{l}_{; \nu}^{\mu \nu}=0$. First we seek a particular retarded solution to Eq. (9). Here it is useful to remove the restriction $x \notin z_{G}(\tau)$, and continue $\delta S_{\mu \nu}$ to the worldline $z_{G}(\tau)$. Clearly a solution to the continued equation also satisfies the original equation (i.e. with the worldline excluded). We choose the simplest continuation by demanding that no additional singularities (e.g. delta functions) are introduced on the worldline i.e., the only singularities on the worldline must be those originating from a local expansion of $S_{\mu \nu}$. Eq. (91) now reads

$$
\square \delta \bar{l}_{\mu \nu}+2 R_{\mu \nu}^{\eta \rho} \delta \bar{l}_{\eta \rho}=-2 \delta S_{\mu \nu}
$$


Here $\square \equiv g^{\alpha \beta} \nabla_{\alpha} \nabla_{\beta}$. We define $\delta \bar{l}_{\mu \nu}$ to be the retarded solution to Eq. (11). Since $\delta S_{\mu \nu}$ diverges only as $r^{-2}$ its retarded solution has a finite contribution originating from the vicinity of $z_{G}(\tau)$.

By definition the retarded solution $\delta \bar{l}_{\mu \nu}$ satisfies Eq. (11). However, we still have to verify that it also satisfies Eq. (9). This equation will be satisfied if $\delta \bar{l}_{\mu \nu}$ satisfies the Lorenz gauge conditions. To investigate this point one can apply the divergence operator to Eq. (111), thereby constructing a differential equation for $\nabla^{\nu} \delta \bar{l}_{\mu \nu}$. The fact that $\nabla^{\nu} \delta \bar{l}_{\mu \nu}=0$ then follows from the properties of the source term of this equation, namely $-2 \nabla^{\nu} \delta S_{\mu \nu}$. Using a perturbation expansion of the Bianchi identities one finds that $\nabla^{\nu} \delta S_{\mu \nu} \equiv 0$ for $x \notin z_{G}(\tau)$. This property together with an analysis of $\nabla^{\nu} \delta S_{\mu \nu}$ as one approaches the worldline reveals that the retarded solution to Eq. (11) satisfies the Lorenz gauge conditions, and therefore it also satisfies Eq. (9) as required. (The full analysis will be given elsewhere [16].)

So far we have constructed a particular solution (8) to Eq. (44). We now construct the general solution $\bar{l}_{\mu \nu}^{G}$ to this equation. Later we shall impose a set of additional requirements on $\bar{l}_{\mu \nu}^{G}$, and thereby obtain a particular solution which is physically meaningful. Since Eq. (44) is valid for $x \notin z_{G}(\tau)$, we find that we can construct a new solution by adding to $\bar{l}_{\mu \nu}$ a potential that satisfies a semi-homogeneous equation ,i.e., a homogeneous equation for $x \notin z_{G}(\tau)$, reading

$$
D_{\mu \nu}\left[\bar{l}^{S H}\right]=0, x \notin z_{G}(\tau) .
$$

The general solution to Eq. (44) is given by $\bar{l}_{\mu \nu}^{G} \equiv \bar{l}_{\mu \nu}^{S H}+\bar{l}_{\mu \nu}$, where $\bar{l}_{\mu \nu}^{S H}$ is the general solution to Eq. (12).

The set of additional requirements to be imposed on $\bar{l}_{\mu \nu}^{G}$ can be expressed as requirements on $\bar{l}_{\mu \nu}^{S H}$. Denoting $\bar{\gamma}_{\mu \nu}$ the semi-homogeneous potential that satisfies these requirements, we express the desired physical solution $\bar{l}_{\mu \nu}^{P}$ as

$$
\bar{l}_{\mu \nu}^{P}=\bar{l}_{\mu \nu}+\bar{\gamma}_{\mu \nu}=\bar{\psi}_{\mu \nu}+\delta \bar{l}_{\mu \nu}+\bar{\gamma}_{\mu \nu} .
$$

We consider the following additional requirements: (i) gauge conditions, (ii) causality requirements, (iii) global boundary conditions, and (iv) boundary conditions as $x \rightarrow z_{G}(\tau)$. (i) We impose Lorenz gauge conditions on $\bar{\gamma}_{\mu \nu}$. Eq. (12) now takes the form

$$
\square \bar{\gamma}_{\mu \nu}+2 R_{\mu \nu}^{\eta \rho} \bar{\gamma}_{\eta \rho}=0, x \notin z_{G}(\tau) .
$$

(ii) We define $\bar{\gamma}_{\mu \nu}$ to be a retarded solution to Eq. (14). Recall that $\delta \bar{l}_{\mu \nu}$ is the retarded solution to Eq. (111), and $\bar{\psi}_{\mu \nu}$ is completely determined by $\bar{h}_{\mu \nu}^{F}$. This construction implies 
that if we prescribe initial data for the metric perturbations on a spacelike hypersurface $\Sigma_{0}$, then the physical solution $\bar{l}_{\mu \nu}^{P}(x)$ at the future of $\Sigma_{0}$ is unaffected by an arbitrary modification of the initial data outside $J^{-}(x) \cap \Sigma_{0}$, and in this sense the second-order solution $\bar{l}_{\mu \nu}^{P}(x)$ is manifestly causal. (iii) We demand that the only source of $\bar{\gamma}_{\mu \nu}$ is the black-hole itself, thus excluding any incoming waves. (iv) Let us consider once more a small but finite mass $\mu$. Here, Eq. (14) is valid at $x \notin S$. Following D'Eath's analysis of FOMP [1] we express a solution to Eq. (14) using a Kirchhoff representation. Denoting $\Sigma_{E}$ the boundary of $S$, we express $\bar{\gamma}_{\mu \nu}$ in terms of the following surface integral

$$
\begin{aligned}
\bar{\gamma}_{\mu \nu}(x)= & -\frac{1}{4 \pi} \int_{\Sigma_{E}(\mu)}\left(G_{\mu \nu \alpha^{\prime} \beta^{\prime}}^{r e t}\left[x \mid x^{\prime}\right] \nabla^{\epsilon^{\prime}} \bar{\gamma}^{\alpha^{\prime} \beta^{\prime}}\left(x^{\prime}\right)\right. \\
& \left.-\bar{\gamma}^{\alpha^{\prime} \beta^{\prime}}\left(x^{\prime}\right) \nabla^{\epsilon^{\prime}} G_{\mu \nu \alpha^{\prime} \beta^{\prime}}^{r e t}\left[x \mid x^{\prime}\right]\right) d \Sigma_{\epsilon^{\prime}} .
\end{aligned}
$$

Here $G_{\mu \nu \alpha^{\prime} \beta^{\prime}}^{r e t}\left[x \mid x^{\prime}\right]$ denotes the retarded Green's function (for its definition and properties see e.g. [11] $), d \Sigma_{\epsilon^{\prime}}$ denotes an outward directed three-surface element on $\Sigma_{E}$. Consider substituting an expansion (in powers of $r$ ) of a given potential $\bar{\gamma}^{\alpha \beta}$ into Eq. (15), and then taking the limit $\mu \rightarrow 0$. Recall that in this limit we have $r_{E}(\mu) \rightarrow 0$, and notice that $d \Sigma_{\epsilon}$ scales as $r_{E}^{2}$. Therefore, only the diverging terms (as $r \rightarrow 0$ ) in the expansion can contribute to $\bar{\gamma}_{\mu \nu}(x)$ in the limit. These divergent boundary conditions for Eq. (14) are obtained from Eq. (13) together with an analysis of the divergent behavior of $\bar{\psi}_{\mu \nu}, \delta \bar{l}_{\mu \nu}$, and $\bar{l}_{\mu \nu}^{P}$ in near $r=0$.

First we expand $\bar{\psi}_{\mu \nu}$ near $r=0$ and obtain $\bar{\psi}_{\mu \nu} \stackrel{*}{=}-\frac{1}{4 r^{2}}\left[2 u_{\mu} u_{\nu}+7 \eta_{\mu \nu}\right]+O\left(r^{0}\right)$. Next we consider $\delta \bar{l}_{\mu \nu}$. Solving Eq. (11) approximately in the vicinity of $z_{G}(\tau)$ reveals that $\delta \bar{l}_{\mu \nu}$ is bounded as $r \rightarrow 0$ [16]. Finally, we expand $\bar{l}_{\mu \nu}^{P}$ using the internal-zone solution, which is discussed next.

We now calculate the desired diverging terms in the expansion of $\bar{l}_{\mu \nu}^{P}$. For this purpose we use the method of matched asymptotic expansions. For simplicity we assume that all the length scales characterizing the background spacetime $\left\{\mathcal{R}_{i}\right\}$ are of the same order of magnitude $\mathcal{R}$. Expanding the full spacetime metric in the internal-zone using the smallness of $r / \mathcal{R}$ and $\mu / \mathcal{R}$ yields

$$
g_{\mu \nu}^{\text {full }}=g_{\mu \nu}^{S c h}+\mathcal{R}^{-1} g_{\mu \nu}^{(1)}+\mathcal{R}^{-2} g_{\mu \nu}^{(2)}+O\left(\mathcal{R}^{-3}\right) .
$$

Here $g_{\mu \nu}^{S c h}$ is the metric of the Schwarzschild black-hole. Recall that in the buffer-zone both Eq. (16) and Eq. (21) are valid, and should therefore match with each other. To match 
the internal-zone metric with the external-zone metric we further expand Eqs. (216) in the buffer-one.

First we consider the internal-zone expansion. The first term in Eq. (16) is the Schwarzschild metric $g_{\mu \nu}^{S c h}$. In isotropic cartesian coordinates this metric takes the form $d s^{2}=-(2 \rho-\mu)^{2}(2 \rho+\mu)^{-2} d t^{2}+\left[1+\mu(2 \rho)^{-1}\right]^{4}\left(d x^{2}+d y^{2}+d z^{2}\right)$, where $\rho^{2}=x^{2}+y^{2}+z^{2}$. In the buffer-zone $\mu \ll \rho$ and therefore the Schwarzschild metric can be approximated with an asymptotic expansion in powers of $\mu \rho^{-1}$ giving

$$
g_{\mu \nu}^{S c h}=\eta_{\mu \nu}+\mu \rho^{-1} H_{\mu \nu}^{1}+\mu^{2} \rho^{-2} H_{\mu \nu}^{2}+O\left(\mu^{3} \rho^{-3}\right),
$$

where $H_{\mu \nu}^{1}=2\left(\eta_{\mu \nu}+2 \delta_{\mu}^{0} \delta_{\nu}^{0}\right), H_{\mu \nu}^{2}=1 / 2\left(3 \eta_{\mu \nu}-\delta_{\mu}^{0} \delta_{\mu \nu}^{0}\right)$. The next term in Eq. (16) is $\mathcal{R}^{-1} g_{\mu \nu}^{(1)}$. In a suitable gauge this term vanishes identically [10].

Next we consider the external-zone expansion (2). Recall that in the buffer-zone $r \ll \mathcal{R}$. We therefore expand the terms in Eq. (2) in powers of $r \mathcal{R}^{-1}$. The first term in Eq. (2) is the background metric $g_{\mu \nu}$. Expressing this metric with Fermi normal coordinates based on the worldline, and expanding it in powers of $r \mathcal{R}^{-1}$ gives $g_{\mu \nu} \stackrel{*}{=} \eta_{\mu \nu}+O\left(\mathcal{R}^{-2} r^{2}\right)$. Expanding the next term in (2) gives $\mu h_{\mu \nu}^{F} \stackrel{*}{=} 2 \mu r^{-1}\left(\eta_{\mu \nu}+2 u_{\mu} u_{\nu}\right)+O\left(\mu \mathcal{R}^{-2} r^{1}\right)$, and expanding the third term $\mu^{2} l_{\mu \nu}^{P}$ gives a sum of a schematic form $\mu^{2} \sum_{i=0}^{\infty} \mathcal{R}^{-i} r^{i-2}$. Recall that here we are only concerned with divergent boundary conditions that are encoded in the $i=0,1$ terms.

First let us consider matching the first three terms in the expansion of the Schwarzschild metric (17), all these terms scale like $\mathcal{R}^{0}$. From the above expansions we find that both $g_{\mu \nu}^{S c h}$ and $g_{\mu \nu}$ have the same leading term $-\eta_{\mu \nu}$. The next term in Eq. (17) is $\mu \rho^{-1} H_{\mu \nu}^{1}$ this term coincides with the leading order expansion of $\mu h_{\mu \nu}^{F}$, where we identify $\rho$ with $r$. The next term in Eq. (17) provide us with the desired $i=0$ term in the expansion of $\mu^{2} l_{\mu \nu}^{P}$. We find that this term is identical to the leading term in the expansion of $\psi_{\mu \nu}$.

Next let us consider matching the terms that scale like $\mathcal{R}^{-1}$. Since $\mathcal{R}^{-1} g_{\mu \nu}^{(1)}=0$ all the terms that scale like $\mathcal{R}^{-1}$ vanish. This conforms with the fact that the expansions of $g_{\mu \nu}$ and $\mu h_{\mu \nu}^{F}$ do not contain terms that scale like $\mathcal{R}^{-1}$. This also implies that in the expansion of $\mu^{2} l_{\mu \nu}^{P}$ the term with the schematic form $\mu^{2} \mathcal{R}^{-1} r^{-1}$ is zero. Combining the $i=0$ and $i=1$ terms in the expansion of $\mu^{2} l_{\mu \nu}^{P}$ we conclude that $\bar{l}_{\mu \nu}^{P} \stackrel{*}{=}-\frac{1}{4 r^{2}}\left[2 u_{\mu} u_{\nu}+7 \eta_{\mu \nu}\right]+O\left(r^{0}\right)$.

Combining the expansions of $\bar{\psi}_{\mu \nu}$ and $\bar{l}_{\mu \nu}^{P}$ near $r=0$, together with the fact that $\delta \bar{l}_{\mu \nu}$ is bounded in the vicinity of the worldline, and using Eq. (13) we find that $\bar{\gamma}_{\mu \nu}$ does not diverge as $x \rightarrow z_{G}(\tau)$. As was previously discussed only divergent boundary conditions (as 
$r \rightarrow 0$ ) can produce non-vanishing contributions to $\bar{\gamma}_{\mu \nu}(x)$. Since we found that $\bar{\gamma}_{\mu \nu}$ does not diverge in the vicinity of the worldline we conclude that $\bar{\gamma}_{\mu \nu} \equiv 0$. By virtue of Eq. (13) we finally conclude that the physical SOMP are given by

$$
\bar{l}_{\mu \nu}^{P}=\bar{\psi}_{\mu \nu}+\delta \bar{l}_{\mu \nu} .
$$

Here $\bar{\psi}_{\mu \nu}$ is given by Eq. (10), and $\delta \bar{l}_{\mu \nu}$ is the retarded solution to Eq. (11). By construction $\bar{l}_{\mu \nu}^{P}$ satisfies the previously mentioned requirements. In particular it is manifestly causal, and it matches with the internal-zone solution. Moreover, Eq. (18) provides a simple covariant prescription for the construction of the SOMP, without any reference to a particular (background) coordinate system. This allows a considerable amount of flexibility in the construction of the SOMP.

\section{Acknowledgments}

I am grateful to Amos Ori and Eric Poisson for numerous valuable discussions. This work was supported in part by the Natural Sciences and Engineering Research Council of Canada, and also in part by The Israel Science Foundation (grant no. 74/02-11.1).

[1] P.D. D'Eath Black Holes Gravitational Interactions (Oxford, Oxford University Press, 1996) Chapt. 3.

[2] Y. Mino, M. Sasaki, and T. Tanaka, Phys. Rev. D 55, 3457 (1997).

[3] T.C. Quinn and R.M. Wald, Phys. Rev. D 56, 3381 (1997).

[4] P. Bender et.al. 1998 LISA - laser interferometer space antenna, Pre-Phase A report, MPQ233 (Max-Planck-Institut für Quantenoptic).

[5] C. Cutler and K.S. Thorne Proceedings of GR16 (Durban, South Africa, 2001); gr-qc/0204090.

[6] L. Barack and C. Cutler, Phys. Rev. D 69, 082005 (2004).

[7] L.M. Burko, Phys. Rev. D 67, 084001 (2003).

[8] S. Detweiler, Class. Quant. Grav. 22, S681 (2005).

[9] E. Rosenthal, Class. Quant. Grav. 22, S859 (2005).

[10] K.S. Thorne and J.B. Hartle, Phys. Rev. D 31, 1815 (1985).

[11] E. Poisson, Living Rev. Rel. 7, 6 (2004); online at http://www.livingreviews.org/lrr-2004-6 
[12] C.W. Misner, K.S. Thorne and J.A. Wheeler, Gravitation $§ 35.13$ (San Francisco, Freeman, 1973).

[13] L. Barack and A. Ori, Phys. Rev. D 64, 124003 (2001).

[14] S. Detweiler and B.F. Whiting, Phys. Rev. D 67, 024025 (2003).

[15] A. Ori, private communication.

[16] E. Rosenthal, in preparation. 